\title{
Extended Rayleigh Model of Bubble Evolution with Material Strength Compared to Detailed Dynamic Simulations
}

\author{
M. E. Glinsky, P. A. Amendt, D. S. Bailey,
}

R. A. London, A. M. Rubenchik, and M. Strauss

This paper was prepared for submittal to the Society of Photo-Optical Instrumentation Engineers BiOS '97 San Jose, California

February 8-14, 1997

March 4, 1997

This is a preprint of a paper intended for publication in a journal or proceedings. Since changes may be made before publication, this preprint is made available with the understanding that it will not be cited or reproduced without the permission of the author. 


\section{DISCLAIMER}

This document was prepared as an account of work sponsored by an agency of the United States Government. Neither the United States Government nor the University of California nor any of their employees, makes any warranty, express or implied, or assumes any legal liability or responsibility for the accuracy, completeness, or usefulness of any information, apparatus, product, or process

disclosed, or represents that its use would not infringe privately owned rights. Reference herein to any specific commercial product, process, or service by trade name, trademark, manufacturer, or otherwise, does not necessarily constitute or imply its endorsement, recommendation, or favoring by the United States Government or the University of California. The views and opinions of authors expressed herein do not necessarily state or reflect those of the United States Government or the University of California, and shall not be used for advertising or product endorsement purposes. 


\title{
Extended Rayleigh Model of Bubble Evolution with Material Strength Compared to Detailed Dynamic Simulations
}

\author{
Michael E. Glinsky, Peter A. Amendt, David S. Bailey, Richard A. London and Alexander M. Rubenchik \\ Lawrence Livermore National Laboratory \\ Livermore, California 94550 \\ Moshe Strauss \\ Nuclear Research Center, Negev \\ Beer Sheva, Israel
}

\begin{abstract}
The validity of an extended Rayleigh model for laser generated bubbles in soft tissue is examined. This model includes surface tension, viscosity, a realistic water equation of state, material strength and failure, stress wave emission and linear growth of interface instabilities. It is compared to detailed dynamic simulations using the computer program LATIS. These simulations include stress wave propagation, a realistic water equation of state, material strength and failure, and viscosity. The extended Rayleigh model and the detailed dynamic simulations are compared using a 1-D spherical geometry with a bubble in the center and using a 2-D cylindrical geometry of a laser fiber immersed in water with a bubble formed at the end of the fiber. Studies are done to test the validity of the material strength and failure, stress wave emission, and the interface instability terms in the extended Rayleigh model. The resulting bubble radii, material damage radii, the emitted stress wave energies, and the size of the interface distortions are compared. Conclusions are made on the validity of the extended Rayleigh model and on possible improvements to this model. The purpose of this study is to investigate the use of the extended Rayleigh model as a substitute for the detailed dynamic simulations when only limited information is needed. It is also is meant to benchmark the detailed dynamic simulations and highlight the relevant physics. It is shown that the extended Rayleigh model executes over 300 times faster on a computer than the detailed dynamic simulations.
\end{abstract}

Keywords: bubble, stress wave, material failure, acoustic radiation, simulation

\section{INTRODUCTION}

There has been recent interest in using the stress caused by the expansion of a small vapor bubble to precisely cut biological tissues. ${ }^{1}$ The bubble is formed either by the absorption of laser light or by an electrical discharge. Such bubbles are similar to cavitation bubbles, whose study has a long rich history, including work in the 1940's, prompted by the U.S. Navy's need to understand the mechanics of cavitation bubbles that were damaging their propellers. ${ }^{2}$ One of the results of this research was an enhanced Rayleigh model for the bubble evolution and stability.

It is important to know the most efficient way to cut a specified amount of material with laser produced bubbles. The high efficiency is needed to minimize the amount of residual heat which can cause unwanted damage. Detailed dynamic simulations can take too long to do comprehensive parameter studies. We were therefore motivated to further extend the Rayleigh model to include material strength and failure and to correctly account for the emitted acoustic energy. The latter because it is a significant sink for energy that would otherwise end up as bubble expansion energy. The advantage of a extended Rayleigh model (ERM) over a more detailed dynamic simulation is speed. The extended Rayleigh model is a second order ODE for the bubble radius. In contrast, the detailed dynamic simulations are a solution of a set of partial differential equations. Although the ERM is more efficient, it does not give information such as the detailed evolution of the stress wave or shock once it is emitted. It also does not take into account any internal structure of the bubble or the more complicated models of material failure. Because of the limitations, it is necessary to compare the ERM to the more detailed dynamic simulations and to verify that the assumptions are valid. 


\section{EXTENDED RAYLEIGH MODEL}

The ordinary differential equation (ODE) describing the temporal evolution of the bubble is based on the work of Rayleigh, Plesset, and Gilmore. ${ }^{2}$ We have modified the equation to correctly account for the partial reflections of stress waves at the surface of the bubble, and material strength and failure. This model assumes that there are two regions: the interior of the bubble and the exterior. The second order ODE for the position of the bubble wall, $R$, is

$$
R \ddot{R}\left(1-\dot{R} / c_{s}\right)+\frac{3}{2} \dot{R}^{2}\left(1-\dot{R} / 3 c_{s}\right)=h\left(1+\dot{R} / c_{s}\right)+F \frac{R}{c_{s}} \dot{h}\left(1-\dot{R} / c_{s}\right),
$$

where

$$
\begin{aligned}
h \equiv \text { enthalpy change between bubble wall and infinity }=\int_{P_{0}}^{P} \frac{d P}{\rho_{s}} \\
F \equiv \frac{\rho_{s}-\rho_{b} \dot{R}\left(\frac{c_{b}-\dot{R}}{c_{s}^{2}-\dot{R}^{2}}\right)}{\rho_{b} \frac{c_{b}-\dot{R}}{c_{s}+\dot{R}}+\rho_{s}} \\
P_{0} \equiv \text { pressure at infinity } \\
c_{s} \equiv \text { sound speed in solid } \\
c_{b} \equiv \text { sound speed in bubble } \\
\rho_{s} \equiv \text { solid density } \\
\rho_{b} \equiv \text { bubble density }
\end{aligned}
$$

The pressure is given by the equation

$$
P=P_{b}-\frac{2 \sigma}{R}-4 \eta \frac{\dot{R}}{R}-Y(\{R(t)\}),
$$

where

$$
\begin{aligned}
& \sigma \equiv \text { surface energy } \\
& \eta \equiv \text { viscosity } \\
& Y \equiv \text { radial stress } \\
& P_{b} \equiv \text { bubble pressure (from equation of state) } .
\end{aligned}
$$

The Kirkwood-Bethe approximation has been made in obtaining this equation. This is an assumption that $h+\dot{R}^{2} / 2$ is propagated by a wave equation with velocity $c_{s}+\dot{R}$ away from the bubble-solid boundary. This approximation should be valid for weak shocks [that is bubble wall Mach number $=\dot{\mathrm{R}} / \mathrm{c}_{\mathrm{s}} \leq O(1)$ ]. One can expect it to break down for strong shocks. Modifications of the propagation velocity to account for the shock propagation is the subject of our future research. We have also added the factor $F$ to account for partial reflection of the stress wave (or weak shock) at the bubble-solid interface. Note that for small Mach number $F=\rho_{s} c_{s} /\left(\rho_{s} c_{s}+\rho_{b} c_{b}\right)$. This shows a complete reflection of the ingoing wave (i.e., $\left.F=1\right)$ for $\rho_{b} c_{b} \ll<\rho_{s} c_{s}$; and a complete transmission of the ingoing wave (i.e., $F=0.5$ ) for $\rho_{b} c_{b}=\rho_{s} c_{s}$.

The material strength and failure has been included by the radial stress term $Y(\{R(t)\})$ which is a function of the complete time history of the bubble wall $\{R(t)\}$. To obtain the expression for this stress it is assumed that the solid is locally perfectly elastic with a shear modulus of $\mu$ and is incompressible. ${ }^{3}$ When the failure stress $Y_{0}$ is exceeded, the material fails completely and the local stress goes to zero. This gives the expression

$$
Y=4 \mu\left(\frac{R-R_{0}}{R_{0}}\right) \frac{\left(R_{0} R^{2}+R_{0} R+R_{0}^{2}\right) R_{0}}{3 R_{d}^{3}},
$$

where 


$$
\begin{aligned}
& R_{0} \equiv \text { initial unstressed radius } \\
& R_{d} \equiv \max \left(R_{0}, \sqrt[3]{2\left|R_{m}^{3}-R_{0}^{3}\right| / 3 \varepsilon_{d}}\right)=\text { radius to which solid has failed } \\
& R_{m} \equiv \text { maximum radius up to time } t \\
& \varepsilon_{d} \equiv Y_{0} / 2 \mu=\text { failure strain }
\end{aligned}
$$

The behavior of the radial stress over a loading cycle is shown in Fig 1 . The solid acts perfectly elastically as the strain of the bubble wall $e=2\left(R-R_{0}\right) / R$ increases. When the failure stress is reached the material starts to fail out to a radius $R_{d}$ so that the stress remains clamped at $Y_{0}$. As the strain is decreased, the material as a whole acts again as a perfectly elastic solid with a reduced shear modulus due to the irreversible material failure.

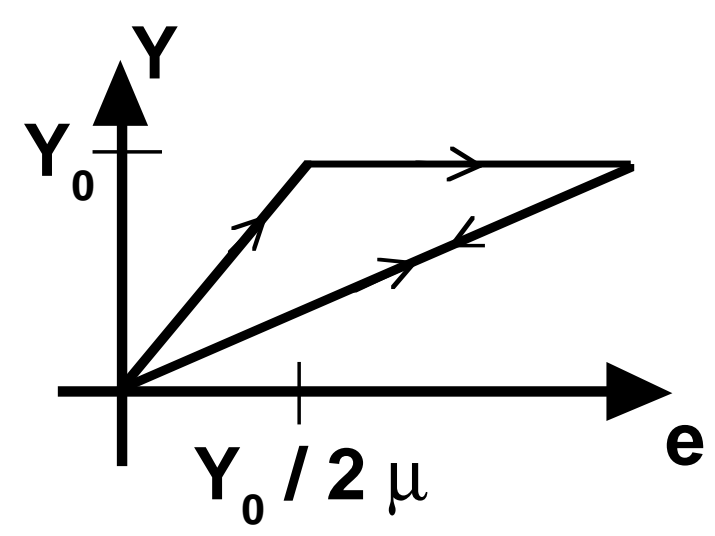

Fig. 1. Stress-strain behavior for material failure model.

The linear growth of the interface instability (commonly called a Rayleigh-Taylor instability) can be determined once $R(t)$ is known. ${ }^{4}$ The following second order ODE determines the perturbation amplitude $\zeta_{l}$ of the spherical harmonic with mode number $l$

$$
\ddot{\zeta}_{l}+3 \dot{R} \dot{\zeta}_{l} / R=A_{l} \zeta_{l}
$$

where

$$
A_{l} \equiv \frac{\left[l(l+1)-(l+1)(l+2) \rho_{b} / \rho_{s}\right] \frac{\ddot{R}}{R}-\left(l^{2}-1\right)(l+2) \frac{l \sigma}{\rho_{s} R^{3}}}{l+(l+1) \rho_{b} / \rho_{s}} .
$$

The eigenvalues of this equation are given by

$$
\gamma_{l \pm}=-3 \dot{R} / 2 R \pm \sqrt{(3 \dot{R} / 2 R)^{2}+A_{l}}
$$

where the evolution scales like $\zeta_{l} \sim \exp \left(\gamma_{l \pm} t\right)$. This implies that the system is stable to interface (shape) instabilities when $\operatorname{Re}\left(\gamma_{l+}\right)<0$. For $\rho_{b}<<\rho_{s}, l>>1$ and neglecting the $\dot{R}$ curvature terms one finds that

$$
\gamma_{l+} \sim \sqrt{l \ddot{R} / R-l^{3} \sigma / \rho_{s} R^{3}} .
$$

The motion will be unstable when $\ddot{R}>0$ (that is when the bubble is near its minimum radius). The effect of surface tension is to stabilize the large $\boldsymbol{l}$ number modes yielding a most unstable mode of 


$$
l_{\max } \sim R \sqrt{\ddot{R} \rho_{s} / \sigma} .
$$

\section{LATIS MODEL}

Enhancements have been made to the computer code, LATIS (an acronym for LAser TISsue) developed at Lawrence Livermore National Laboratory. ${ }^{5}$ In addition to the physics that was previously in the code (laser propagation, laser energy deposition, hydrodynamics, atomic physics, radiation absorption, radiation emission, radiation transport, and thermal transport), we have added material strength and failure.

There are three equations that are used to advance the velocities, strains, and then the stresses in succession. The first is the dynamic equation (a generalization of the fluid momentum equation) given by

$$
\rho \dot{\overrightarrow{\mathrm{v}}}=\nabla \bullet(-P \vec{I}+\vec{S})
$$

where

$$
\begin{aligned}
& \rho \dot{\mathrm{v}}_{r}=-\frac{\partial}{\partial r}\left(P-S_{r r}\right)+\frac{\partial}{\partial z} S_{r z}+\frac{1}{r}\left(2 S_{r r}+S_{z z}\right) \\
& \rho \dot{\mathrm{v}}_{z}=-\frac{\partial}{\partial z}\left(P-S_{z z}\right)+\frac{\partial}{\partial r} S_{r z}+\frac{1}{r} S_{r z}
\end{aligned}
$$

and $P=$ pressure, $\vec{S}=$ deviatoric stress tensor, $\vec{I}=$ identity tensor, $\overrightarrow{\mathrm{v}}=$ velocity, and $\rho=$ density. Two-dimensional cylindrical coordinates, $r$ and $z$, are used. The second is the kinematic equation (a generalized equation of continuity) given by

$$
\frac{\dot{\theta}}{3} \vec{I}+\dot{\vec{e}}=\dot{\vec{\varepsilon}}(\overrightarrow{\mathrm{v}}, \nabla \overrightarrow{\mathrm{v}})
$$

where

$$
\begin{aligned}
& \dot{\theta}=\frac{\partial \mathrm{v}_{r}}{\partial r}+\frac{\partial \mathrm{v}_{z}}{\partial z}+\frac{\mathrm{v}_{r}}{r} \\
& \dot{e}_{r r}=\frac{1}{3}\left(2 \frac{\partial \mathrm{v}_{r}}{\partial r}-\frac{\partial \mathrm{v}_{z}}{\partial z}-\frac{\mathrm{v}_{r}}{r}\right) \\
& \dot{e}_{z z}=\frac{1}{3}\left(2 \frac{\partial \mathrm{v}_{z}}{\partial z}-\frac{\partial \mathrm{v}_{r}}{\partial r}-\frac{\mathrm{v}_{r}}{r}\right), \\
& \dot{e}_{r z}=\frac{1}{2}\left(\frac{\partial \mathrm{v}_{r}}{\partial z}+\frac{\partial \mathrm{v}_{z}}{\partial r}\right)
\end{aligned}
$$

$\vec{e}=$ deviatoric strain tensor, and $\theta=$ fractional volume change. The third equation is a generalization of Hooke's law given by

$$
-\dot{P} \ddot{I}+\dot{\vec{S}}=K\left(\dot{\theta}-\dot{\theta}_{i n}\right)+2 \mu\left(\dot{\vec{e}}-\dot{\vec{e}}_{i n}\right)+\dot{\vec{S}}_{\text {rot }},
$$

where 


$$
\begin{aligned}
& \dot{P}=-K\left(\dot{\theta}-\dot{\theta}_{i n}\right) \\
& \dot{S}_{r r}=2 \mu\left[\dot{e}_{r r}-\left(\dot{e}_{r r}\right)_{i n}\right]+2 S_{r z} \omega_{r z} \\
& \dot{S}_{z z}=2 \mu\left[\dot{e}_{z z}-\left(\dot{e}_{r r}\right)_{i n}\right]-2 S_{r z} \omega_{r z} \\
& \dot{S}_{r z}=2 \mu\left[\dot{e}_{r z}-\left(\dot{e}_{r r}\right)_{i n}\right]+2\left(S_{z z}-S_{r r}\right) \omega_{r z}
\end{aligned}
$$

where $K=$ bulk modulus, $\mu$ =shear modulus, $\theta_{i n}=$ inelastic volume change, $\vec{e}_{i n}=$ inelastic strain tensor and

$$
\omega_{r z}=\frac{1}{2}\left(\frac{\partial \mathrm{v}_{r}}{\partial z}-\frac{\partial \mathrm{v}_{z}}{\partial r}\right)=r z \text { rotation frequency }
$$

The values of the inelastic changes to the strains and volume are chosen such that the pressure relaxes to the minimum pressure $P_{m}$ if it is less than $P_{m}$ and to zero if it is greater than zero, and that the deviatoric stress invariant

$$
Y=\sqrt{2 \vec{S}: \vec{S} / 3}
$$

relaxes to its limit $Y_{e}\left(P_{e}\right)$ for values greater than $Y_{e}\left(P_{e}\right)$, where the effective pressure is

$$
\begin{aligned}
& P_{e}=P-\sqrt[3]{|\vec{S}| / 16} \\
& \vec{S}: \vec{S}=2\left(S_{r r}^{2}+S_{z z}^{2}+S_{r z}^{2}+S_{z z} S_{r r}\right) \text { and } \\
& |\vec{S}|=\left(S_{r r}+S_{z z}\right)\left(S_{r z}^{2}-S_{r r} S_{z z}\right) .
\end{aligned}
$$

The yield surface $Y_{e}\left(P_{e}\right)$ used is shown schematically in Fig. 2.



Fig. 2. Yield surface, $Y_{e}\left(P_{e}\right)$. 
The inelastic change $\theta_{\text {in }}$ is limited so that the inelastic volume, $V_{i n}$, will be between zero and the total volume, $V$. All the deviatoric stresses are scaled by the same factor so that $Y$ will have the desired value.

The damage index, $\varepsilon_{D}$, is calculated as

$$
\varepsilon_{D}=\min \left(1, \frac{e_{p}}{e_{0}}+\frac{\theta_{i n}}{\theta_{0}}\right),
$$

where $e_{0}$ is the shear strain threshold and $\theta_{0}$ is the tensile strain threshold. These thresholds are the critical points where the disconnected flaws (such as voids or cracks) coalesce into a large one and the material "breaks". The plastic strain is defined as

$$
e_{p} \equiv \int\left|d e_{i n}\right|
$$

where

$$
e_{i n}=\sqrt{2 \vec{e}_{i n}: \vec{e}_{i n} / 3} \text {. }
$$

The index for tensile failure is $\theta_{i n}$ - the maximum percentage void volume, $\max \left(V_{i n} / V\right)$ if the tensile damage is not allowed to heal, and $V_{i n} / V$ if the tensile damage is allowed to heal. The density used to calculate the pressure is taken to be the mass in the zone divided by the volume of the cell that is not void, $V-V_{i n}$.

The effect of the damage is to decrease both the minimum pressure $P_{m}$ and the shear yield limit $Y_{e o}$ to $P_{m o}\left(1-\varepsilon_{D}\right)$ and $Y_{\text {eoo }}\left(1-\varepsilon_{D}\right)$ respectively. Here $P_{m o}$ and $Y_{\text {eoo }}$ are constants that characterize the nucleation of the microscopic defects which cause the material failure. This leaves one with the following expressions for the time derivatives for the inelastic strains

$$
\begin{aligned}
& -K \dot{\theta}_{i n}= \begin{cases}\frac{1}{\tau}[P] & \text { if } P>0 \\
0 & \text { if } 0>P<P_{m} \\
\frac{1}{\tau}\left[P-P_{m}\right] & \text { if } P<P_{m}\end{cases} \\
& \dot{\vec{e}}_{i n}= \begin{cases}\frac{\vec{S}}{\tau Y}\left[Y-Y_{e}\right] & \text { if } Y>Y_{e} \\
0 & \text { if } Y<Y_{e}\end{cases}
\end{aligned}
$$

where the relaxation time is governed by the equation

$$
\frac{d \tau}{d t}= \begin{cases}-\frac{1}{\tau_{o}}[\tau] & \text { if } \varepsilon_{D}=1 \\ -\frac{1}{\tau_{o}}\left[\tau-\tau_{o}\right] & \text { if } \varepsilon_{D}<1\end{cases}
$$

and the basic relaxation time is

$$
\tau_{o}=\max \left(\frac{\text { zone area }}{A c_{s} \min (\Delta r, \Delta z)}, \tau_{o o}\right)
$$

The first term is the sound transit time across the zone and prevents an instability in the numerical integration of the equations of motion. The second term is a zonal user supplied time that accounts in an empirical way for the rate dependence of the material failure. The relaxation of $\tau$ from $\tau_{o}$ to 0 when $\varepsilon_{D}=1$ helps model the coalescence of voids. The time 
constant should be chosen to be the characteristic time for the disconnected flaws to grow from the size on which they are nucleated to the size of the inter-flaw spacing.

The bulk modulus is derived from the EOS. The shear modulus is supplied by the user. Two different EOS's for water were used in this work. The first is a table calculated using QEOS. ${ }^{6}$ This table is valid over a large range in both temperature and density but is not very accurate around the vapor dome. The second EOS was based on the NBS steam tables. Although this table is very accurate around the vapor dome it has a limited range of validity. ${ }^{7}$

\section{RESULTS OF STRESS WAVE EMISSION SIMULATIONS}

A 1-D spherical simulation is used to compare the stress wave emission of the extended Rayleigh model to the detailed dynamic simulations. This geometry is shown in Fig. 3. A sphere of water at liquid density and pressure of 10 bar is instantaneously heated up to a temperature of $T_{0 b}$. The QEOS equation-of-state is used for the water and there is no surface tension or viscosity. The radius as a function of time is monitored as well as the energy that is emitted during the initial expansion and during the first collapse. The results are shown in Figs. 4 and 5. The temporal histories of the bubble radii compare quite well. Both models show no bounce of the bubble for an initial temperature of $200^{\circ} \mathrm{C}$, only one bounce for $300^{\circ} \mathrm{C}$, and many bounces for $400^{\circ} \mathrm{C}$ and $500^{\circ} \mathrm{C}$. The maximum bubble radius for the first expansion for the extended Rayleigh model is $20 \%$ less than for the LATIS simulations, see Fig. 5a, but there is the same trend of increase of the maximum radius as the bubble temperature is increased. The discrepancy in the bubble radius disappears for the second expansion. Both the magnitude and the functional form of the maximum bubble radius match quite well. The efficiency of acoustic radiation during the initial expansion and during the first collapse, Fig. 5b, show the same trends as the maximum bubble radius - there is more energy radiated by the extended Rayleigh model during the first expansion but less during the first collapse.



Fig. 3. Simulation geometry. 
(a)

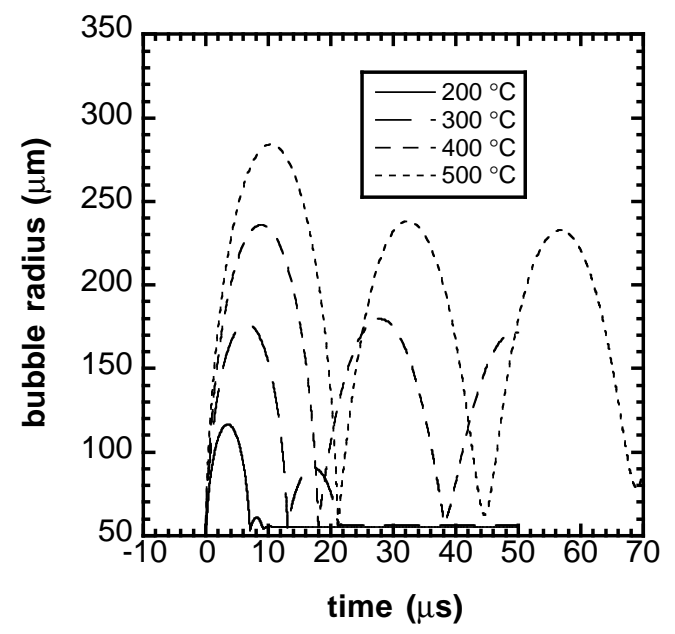

(b)

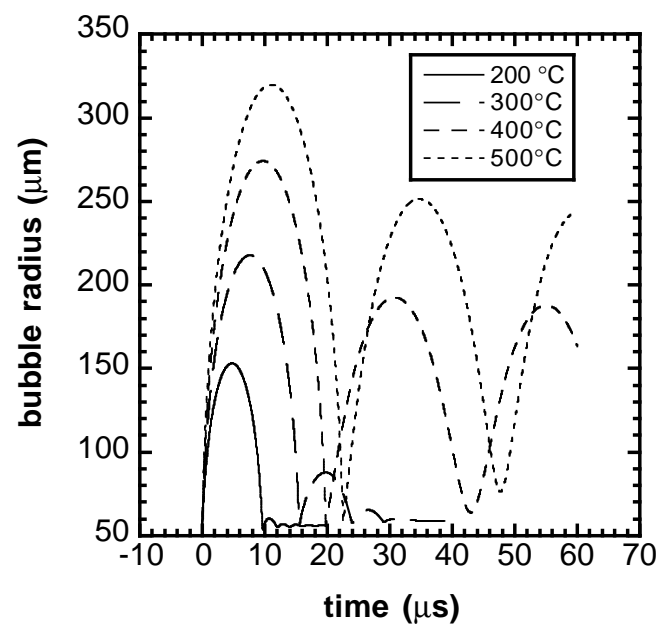

Fig. 4. Bubble radius as a function of time for (a) the ERM and (b) for LATIS.

(a)

(b)
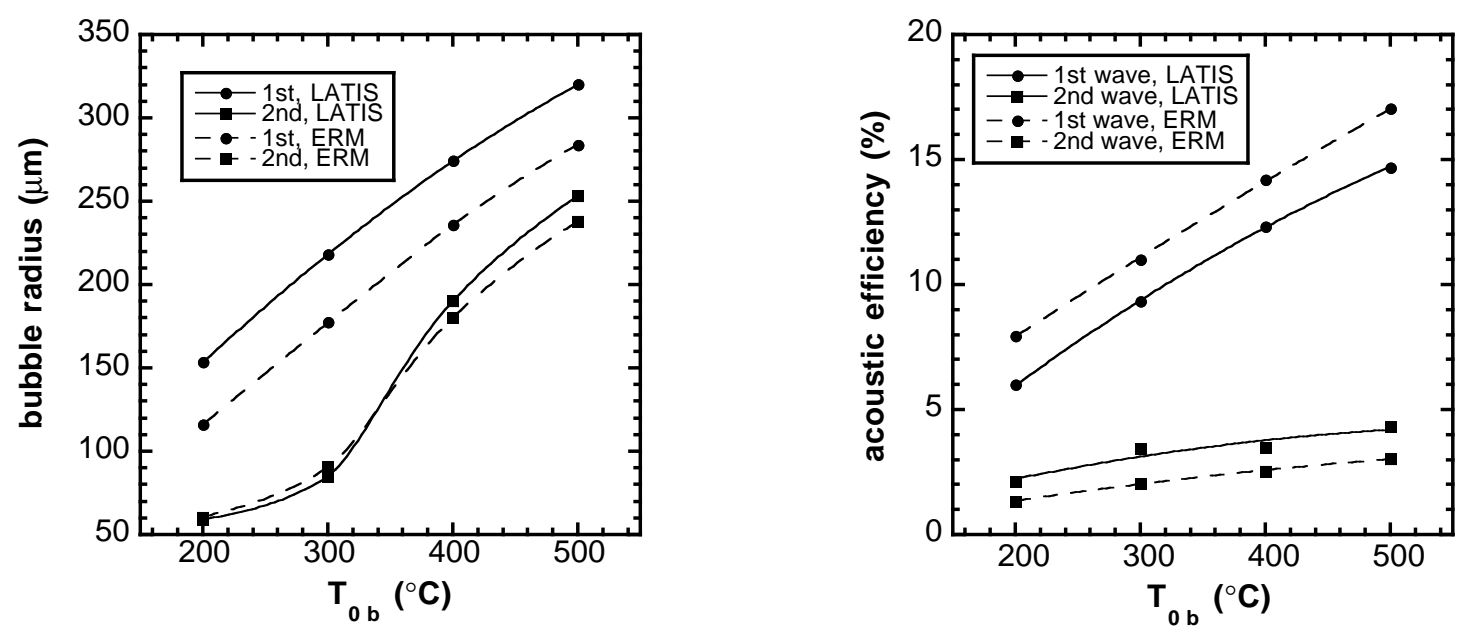

Fig. 5. (a) Maximum bubble radius as a function of initial bubble temperature for the first and second expansion for the extended Rayleigh model (ERM) and LATIS; and (b) the acoustic efficiency as a function of the peak bubble temperature for the first and second emission of acoustic waves. The efficiency is defined as the energy emitted into the acoustic wave divided by the thermal energy required to heat the bubble up to its initial temperature. 


\section{RESULTS OF MATERIAL FAILURE SIMULATIONS}

The material strength and failure term in the ERM is tested using the same 1-D spherical geometry shown in Fig. 3. The initial bubble temperature used is $400^{\circ} \mathrm{C}$ for all the simulations in this section. The EOS is based on the NBS steam tables. The first case is with no material strength or failure. The ambient pressure $P_{0}$ is 10 bar. The results are shown in Fig. 6. The agreement between the ERM and LATIS is good. For the second case, the ambient pressure is reduced to $P_{0}=1$ bar, the shear modulus $\mu$ is set to 300 bar, the shear failure stress $Y_{0}$ to 10 bar and the shear failure strain $e_{0}$ to $8 \times 10^{-5}$. These parameters model a perfectly elastic material which fails immediately and completely when the failure stress is exceeded. The results for the two models are shown in Fig. 7. Both the results for radius of the bubble and for the maximum radius to which the material has completely failed agree well. Also note that the maximum bubble radius is approximately equal for the first two cases. The material strength and failure has acted as an effective ambient pressure of 10 bar. For the final case, the complete failure is delayed by using a failure strain $e_{0}$ of 0.24 . This is done only for a LATIS simulation. There is no way to do the equivalent simulation using the ERM. Figure 8 displays three curves giving the results of this simulation. The first is the bubble radius. The maximum radius is reduced $30 \%$ by delayed coalescence which has raised the effective applied pressure above 10 bar. The second curve is the radius to which the material has completely failed. The third curve delineates the dividing line between the region of partial failure and no failure. Fig. 9 shows examples of typical stressstrain curves for the three regions shown in Fig. 8. The first is that of a perfectly elastic stress-strain curve (undamaged material). The second is that of material that has started to undergo failure but has not yet totally failed. The final curve is that of material that has totally failed and will no longer support any stress. 



Fig. 6. Bubble radius as a function of time for (a) the extended Rayleigh model and (b) for LATIS. $P_{0}=10$ bar, $Y_{0}=0, \mu=0$. 



Fig. 7. Bubble radius as a function of time for (a) the extended Rayleigh model and (b) for LATIS. $P_{0}=1$ bar, $Y_{0}=10$ bar, $\mu=300$ bar, and $e_{0}=8 \times 10^{-5}$. Solid line is the bubble radius. Dotted line is the maximum radius to which the material has failed. 


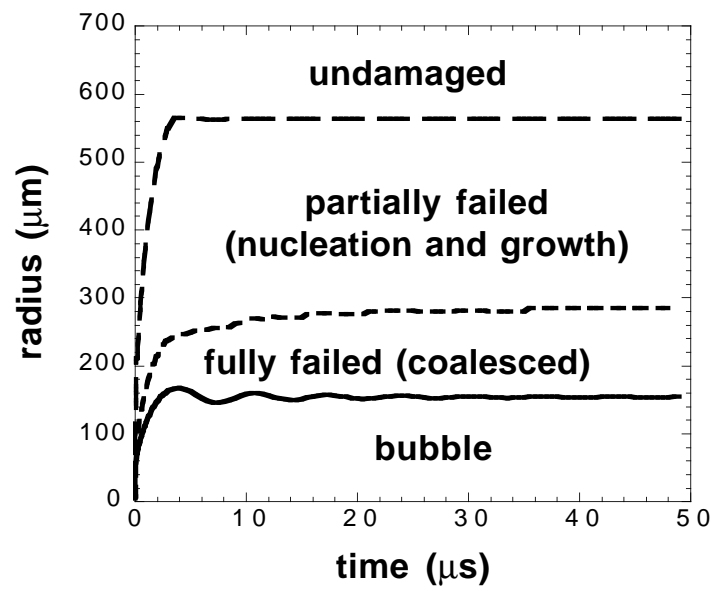

Fig. 8. Bubble radius as a function of time for LATIS. $P_{0}=1$ bar, $Y_{0}=$ $10 \mathrm{bar}, \mu=300 \mathrm{bar}$, and $e_{0}=0.24$. Solid line is the bubble radius. Dotted line in the maximum radius out to which the material has totally failed. Dashed line is the radius out to which there is some

failure.

(a)



(b)

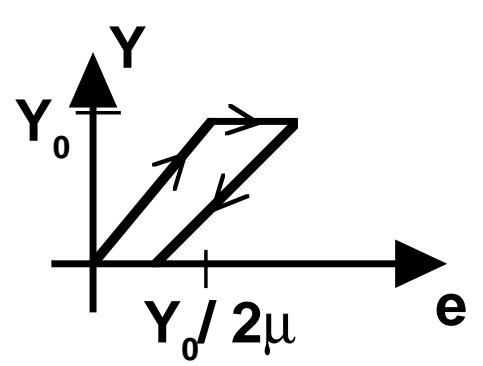

(c)

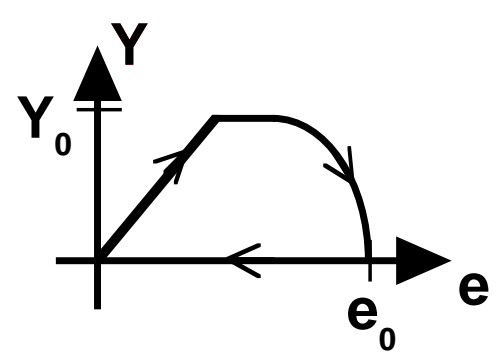

Fig. 9. Examples of stress-strain relationship for (a) perfectly elastic or undamaged material, (b) material which has partially failed and (c) material which has totally failed.

The next three figures, Figs. 10 thru 12, show the details of the evolution of the of the three LATIS simulations just discussed. Three quantities are displayed. The first is three times the density. The factor of three is chosen for convenience of display. The other two quantities are the pressure $P$ and the negation of the radial stress $-S_{r r}$ plotted as the logarithm base 10 of the quantities. Negative quantities correspond to negative values of the quantities. The first case, Fig. 10, shows the pressure wave moving away from the bubble. It leaves behind the pressure profile predicted by the Rayleigh model. Note the small shelf in density on the edge of the bubble at $0.2 \mu \mathrm{s}$. This shelf could act as an acoustic anti-reflective coating causing more stress energy to enter the bubble and be available for bubble motion. This may be responsible for the larger bubble seen in the LATIS simulations of Fig. 4. The second case demonstrates the effect of material strength and failure. As the acoustic wave moves away from the bubble, it has a much greater pressure than radial stress. This is because of the small shear modulus, 300 bar, compared to the bulk modulus of water, $22 \mathrm{kbar}$. The radial shear stress is still enough in this wave to fail the material out to $200 \mu \mathrm{m}$ as evidenced by the zero value of the radial stress out to this radius at $0.2 \mu$ s. For longer 
times the stress builds up to the failure stress of 10 bar as one approaches the bubble. At this point the material can no longer support radial stress since it fails and the stress is transferred to a pressure of 10 bar which is communicated to the bubble wall (see the plot at $9.5 \mu \mathrm{s}$ ). Also note that the radial stress is equivalent to the magnitude of the pressure for the latter time bubble evolution seen in Fig. 11c. The radius of damaged material is extended to almost $1 \mathrm{~mm}$ by the bubble motion. The final case, Fig. 12, demonstrates the effect of gradual failure. The stress pulse propagates out of the problem causing some degree of damage to a $250 \mu \mathrm{m}$ radius. This can be noted by the radial stress being clamped at 10 bar in Fig. 12b. During the longer time bubble evolution, Fig. 12c, one can see the radial stress build up to a maximum value of 10 bar as the bubble wall is approached from large radii. As one further approaches the bubble wall this stress decreases to zero as the material totally fails and the stress is transferred to a pressure of 20 bar that is communicated to the bubble wall. It is this increased pressure which is responsible for the decreased bubble radius.

(a)

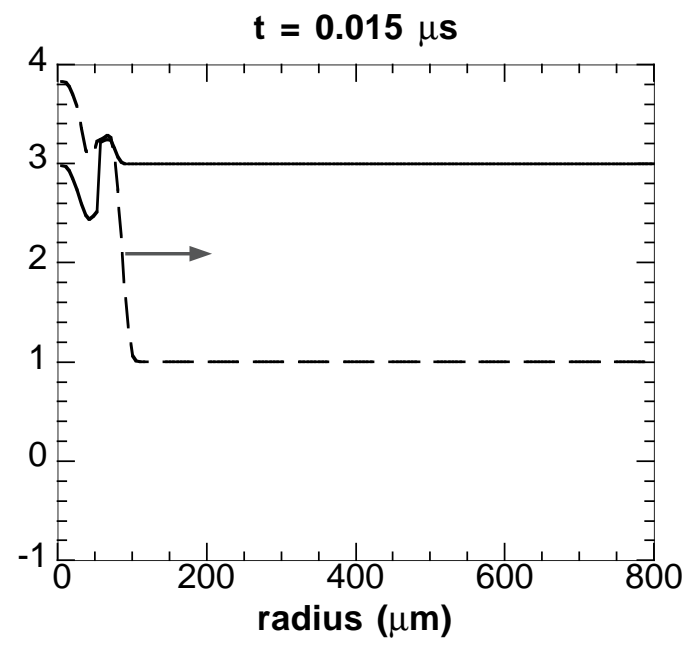

(c)

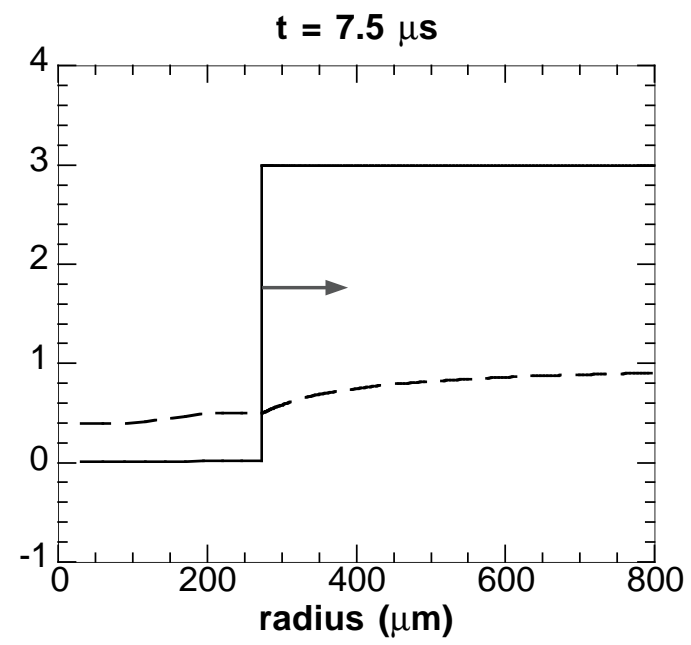

(b)

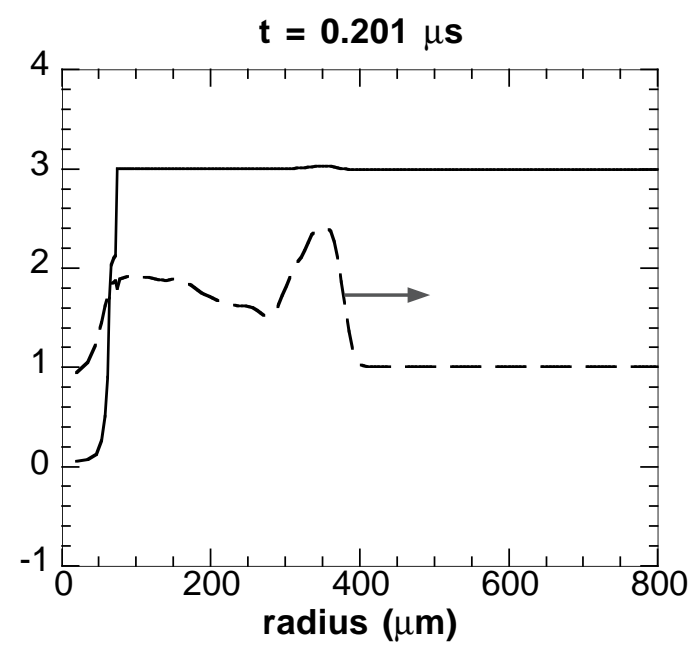

(d)



Fig. 10. Density ( $\rho$, solid line), pressure ( $P$, dashed line) versus

radius. Density is multiplied by three with units of $\mathrm{gm} / \mathrm{cc}$. The pressure is displayed as $\log _{10}(P)$, where $P$ is in bar. Each panel is a snapshot of the time indicated. The arrows indicate direction of motion. The material parameters are: $P_{0}=10 \mathrm{bar}, \mu=0, Y_{0}=0$. 
(a)

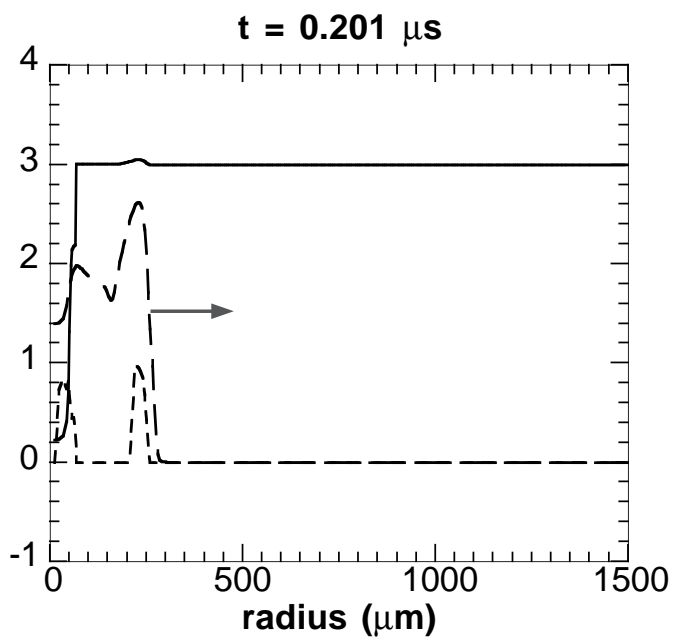

(c)

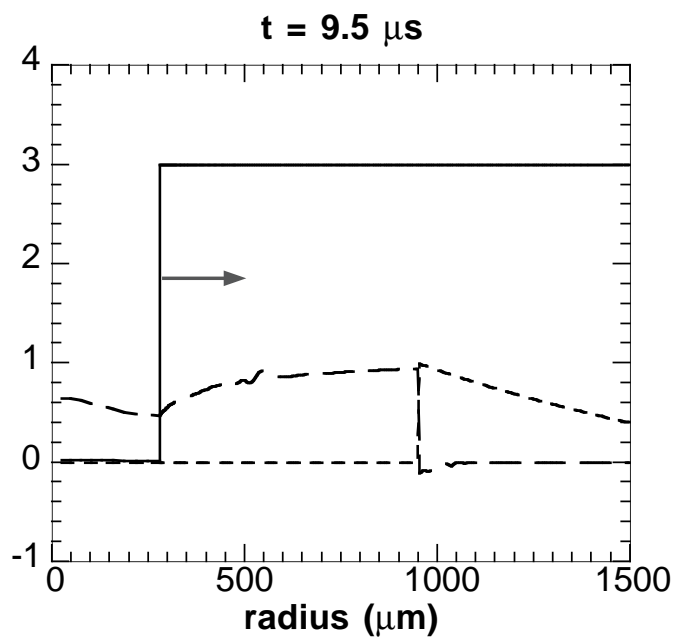

(b)

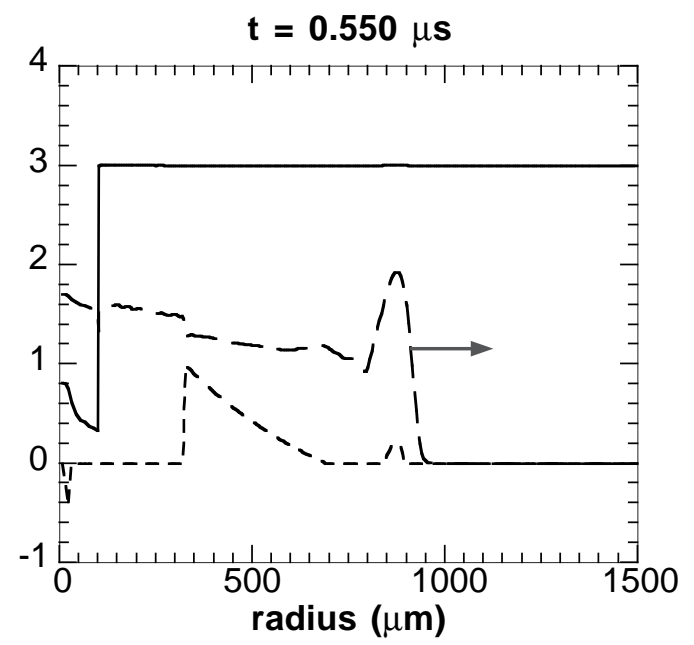

(d)

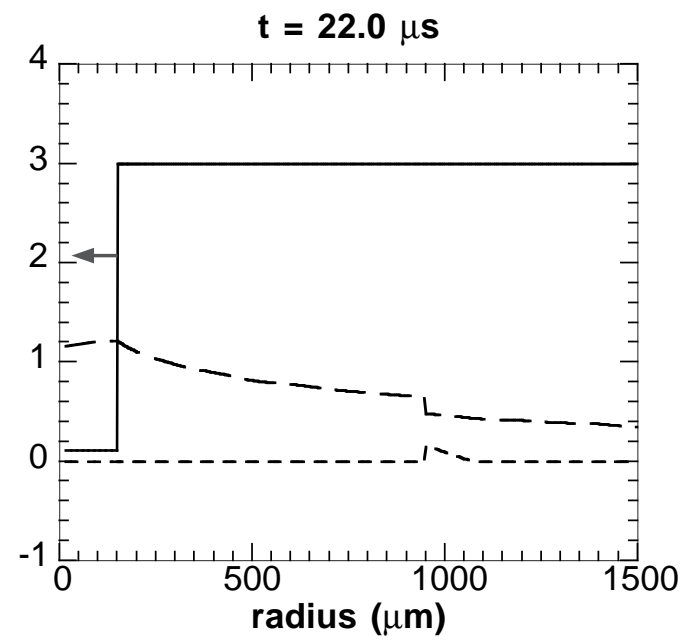

Fig. 11. Density ( $\rho$, solid line), pressure ( $P$, dashed line), and radial stress ( $-S_{r r}$, dotted line) versus radius. Density is multiplied by three with units of $\mathrm{gm} / \mathrm{cc}$. The pressure is displayed as $\log _{10}(P)$, where $P$ is in bar. The radial stress is displayed as $\log _{10}\left(-S_{r r}\right)$, where $S_{r r}$ is in bar. Each panel is a snapshot of the time indicated. The arrows indicate direction of motion. The material parameters are: $P_{0}=1$ bar, $\mu=300$ bar, $Y_{0}=10$ bar, $e_{0}=8 \times 10^{-5}$. 
(a)



(c)



(b)



(d)

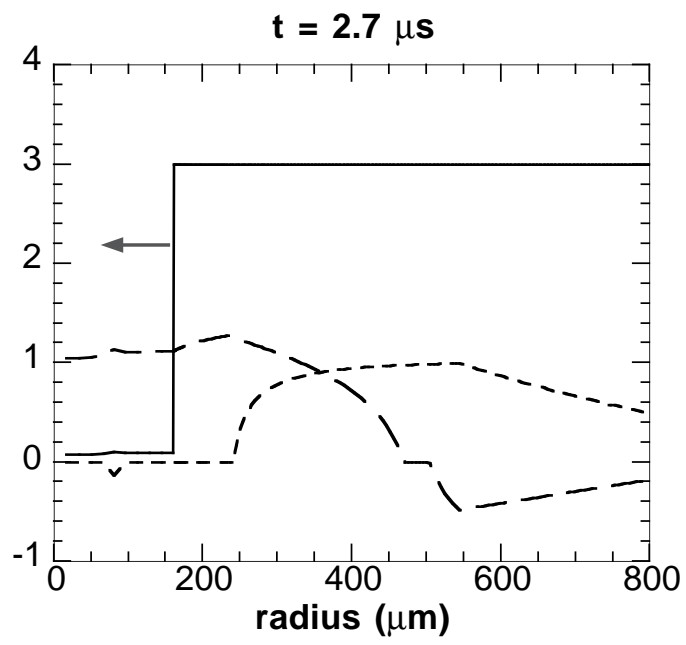

Fig. 12. Density ( $\rho$, solid line), pressure ( $P$, dashed line), and radial stress $\left(-S_{r r}\right.$, dotted line) versus radius. Density is multiplied by three with units of $\mathrm{gm} / \mathrm{cc}$. The pressure is displayed as $\log _{10}(P)$, where $P$ is in bar. The radial stress is displayed as $\log _{10}\left(-S_{r r}\right)$, where $S_{r r}$ is in

bar. Each panel is a snapshot of the time indicated. The arrows indicate direction of motion. The material parameters are: $P_{0}=1$ bar, $\mu=300$ bar, $Y_{0}=10$ bar, $e_{0}=0.24$.

\section{RESULTS OF INTERFACE INSTABILITY SIMULATIONS}

The growth of interface instabilities was studied using the same geometry shown in Fig. 3. The ambient pressure $P_{0}$ was $1 \mathrm{bar}$, the initial bubble temperature was $500^{\circ} \mathrm{C}$, the surface tension $\sigma$ was $70 \mathrm{erg} / \mathrm{cm}^{2}$, and the equation of state was QEOS. Without instability, the bubble bounces more than 10 times. The growth of the instabilities is shown in Fig. 13. The $l=10$ mode grows more than a decade during each collapse of the bubble. The $l=100$ mode is stabilized by the surface 
tension which resists surface perturbation. The behavior of the instability as a function of mode number is demonstrated in Fig. 13b. The maximum instability is for $l=10$ as predicted by Eq. 8 .

The instability model in the ERM is benchmarked qualitatively against a 2-D LATIS simulation. ${ }^{8}$ The geometry for the ERM is shown in Fig. 14. Outside of a $50 \mu \mathrm{m}$ hard core, $0.312 \mathrm{~J}$ of energy are deposited into a $12 \mu \mathrm{m}$ shell. This geometry reproduces both the initial surface area and volume of the $2-\mathrm{D}$ calculation. The size of the $l=1$ mode is shown to grow by more than a decade during the collapse in Fig. 15. This corresponds to the Rayleigh-Taylor bubble and spike behavior seen in the 2-D simulation (Fig. 16b). In this LATIS simulation, $0.312 \mathrm{~J}$ of energy is deposited in a $12 \mu \mathrm{m}$ layer at the end of the laser fiber. The presence of the fiber will seed an $l=1$ mode with an initial perturbation of at least a few percent. This grows into a classic bubble and spike ${ }^{9}$ with Kelvin-Helmhotz rolloff (a shear instability).

(a)

(b)


Fig. 13. Growth of the interface instability. Shown is the size of the mode normalized by its initial amplitude $\zeta_{l} / \zeta_{l 0}$. (a) size of modes versus time. (b) Amplitude of mode at $\mathrm{t}=100 \mu$ s versus mode number. 


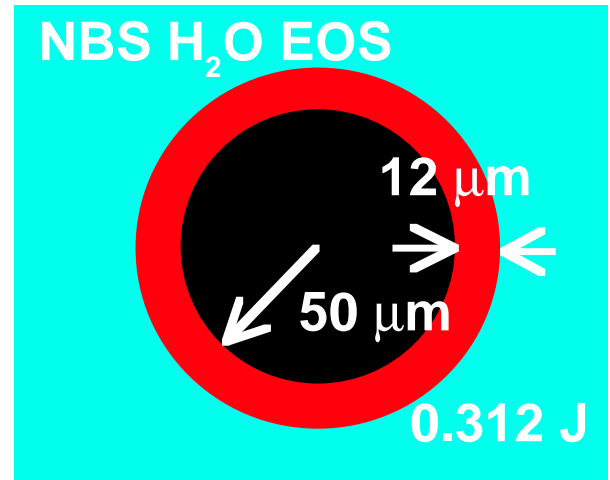

Fig. 14. Geometry used for the ERM to compare to the 2-D LATIS simulations.

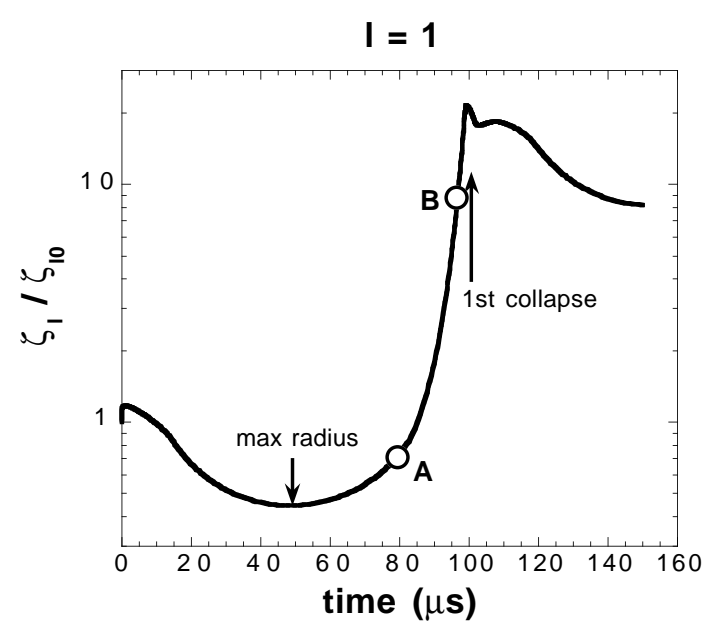

Fig. 15. Growth of the interface instability as a function of time as predicted by the ERM. This is for the geometry shown in Fig. 14. Point A corresponds to the 2-D LATIS simulation in Fig. 16a. Point $\mathrm{B}$ corresponds to the 2-D LATIS simulation in Fig. 16b. 
(a)

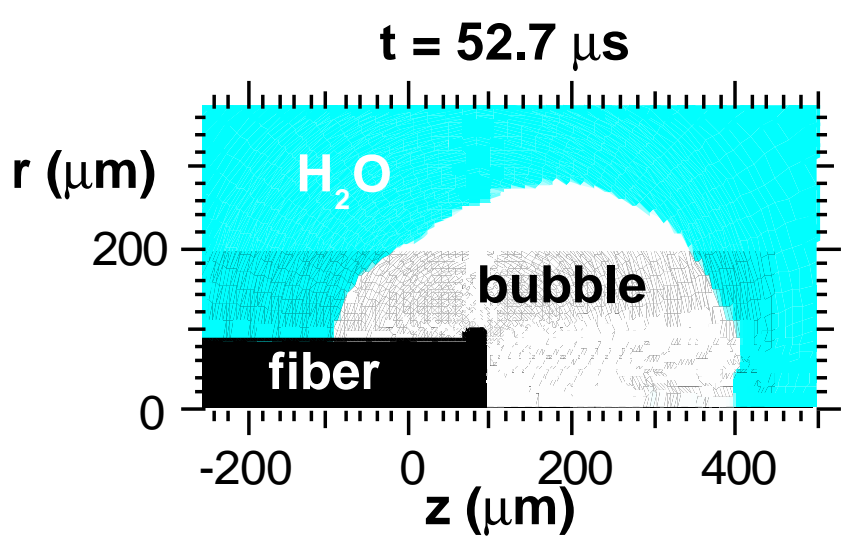

(b)

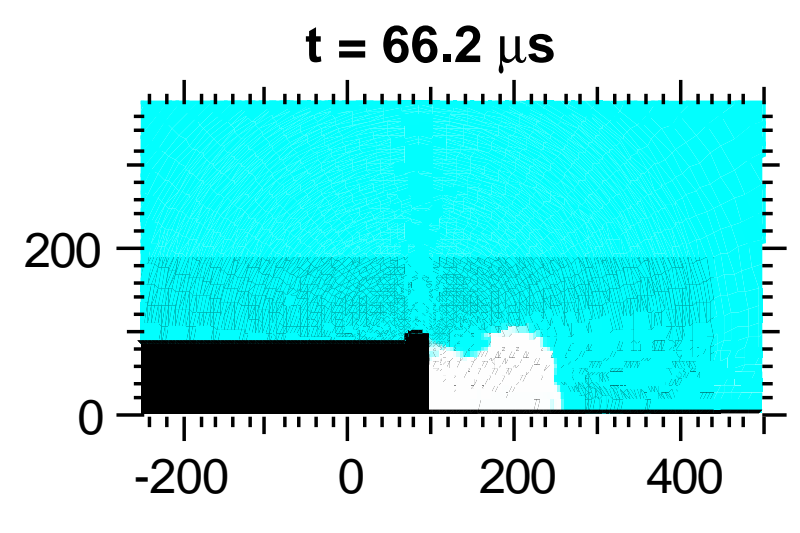

Fig. 16. 2-D LATIS simulation of bubble evolution displaying a $l=1$, bubble and spike instability.

\section{DISCUSSIONS AND CONCLUSIONS}

Three components of the physics contained in the ERM of bubble evolution were benchmarked against LATIS simulations - acoustic radiation, material strength and failure, and interface instability. Good agreement was found for all three. It was also found that taking into account partial reflection of the acoustic wave at the bubble interface is important to find agreement between the ERM and LATIS. Material strength and failure was found to act as an increased ambient pressure, where the effective pressure is the shear failure stress of the material. This is manifested as smaller maximum bubble radii and shorter bubble oscillation periods. The bubble was found to be significantly unstable to interface instabilities on collapse - the magnitude of the oscillation growing a decade per collapse. All modes up to $l=10$ were found to be significantly unstable. There are two important seeds for these instabilities. The first is the laser fiber which will seed a significant $l=1$ mode of at least a few percent in initial amplitude. The second is the presence of an material interface such as a vessel wall. The reflection of the acoustic radiation off of this interface and the subsequent imprint of this wave on the bubble motion when it impinges on the vessel wall would be expected to seed a significant $l=2$ mode. Since these modes will grow about a decade per collapse it is unlikely that the bubble will be able to bounce more than once as a well defined spherical bubble. It was found that the ERM model executed 300 times faster than LATIS on equivalent computers, shortening the time to run a simulation from days to minutes. The extended Rayleigh model with measured physical parameters can now be used to design medical therapies. It enables quick exploration of design parameter space to determine optimal treatment protocols.

\section{ACKNOWLEDGMENTS}

This work was performed under the auspices of the U.S. Department of Energy by the Lawrence Livermore National Laboratory under contract W-7405-ENG-48. One of the authors (MEG) would like to acknowledge the support of a U.S. Department of Energy Distinguished Postdoctoral Fellowship.

\section{REFERENCES}

1. K.W. Gregory, "Laser thrombolysis," Interventional Cardiology, E.J. Topol, editor, W.B. Sanders \& Co., Vol. 2, p. 892, 1994; M. Strauss et al., "Computational modeling of laser thrombolysis for stroke treatment", Proceedings of Lasers in Surgery: Advanced Characterization, Therapeutics, and Systems VI, Vol. 2671, pp. 11-21, 1996; D. Palanker et al., "Electric discharge induced cavitation", Proceedings of Laser Tissue Interaction VIII, Vol. 2975, 1997; A. Vogel et al., "Mechanisms of intraocular photodisruption with picosecond and nanosecond laser pulses", Lasers in Surgery and Medicine, 
Vol. 15, pp. 32-43, 1994; A. Vogel et al., "Minimization of cavitation effects in pulsed laser ablation illustrated on laser angioplasty", Appl. Physics B, Vol. 62, pp. 173-182, 1996; A. Vogel et al., "Shock wave emission and cavitation bubble generation by picosecond and nanosecond optical breakdown in water", J. Acoust. Soc. Am., Vol. 100, pp. 1-18.

2. Lord Rayleigh, "On the pressure developed in a liquid on the collapse of a spherical cavity", Philos. Mag., Vol. 34, p. 94, 1917; M. Plesset, "The dynamics of cavitation bubbles", J. Appl. Mech., Vol. 16, p. 277, 1949; L. Trilling, "The collapse and rebound of a gas bubble", J. Appl. Mech., Vol. 23, pp. 14-17, 1952; R. Hickling and M.S. Plesset, "Collapse and rebound of a spherical bubble in water", Phys. Fluids, Vol. 7, pp 7-14, 1964; F.R. Gilmore, "The growth and collapse of a spherical bubble in a visco compressible Liquid", Calif. Inst. of Tech. Hydrodyn. Lab. Rep. 26-4, 1950. R.T. Knapp, J.W. Daily and F.G. Hammitt, Cavitation, McGraw-Hill, pp. 95-151, 1970.

3. L.D. Landau and E.M. Lifshitz, Theory of Elasticity, Vol. 7, 2nd edition, Pergamon Press Ltd., pp. 20-21, 1970.

4. M.S. Plesset, "On the stability of fluid flows with spherical symmetry", J. Appl. Phys., Vol. 25, pp. 96-98, 1954; S. Chandrasekhar, Hydrodynamic and Hydromagnetic Stability, Oxford University Press, Ch. 10, 1968; Lord Rayleigh, Scientific Papers, Cambridge University press, Vol. II, p. 200, 1900; G.I. Taylor, Proc R. Soc. London Ser. A, Vol. 201, p. 192, 1950.

5. M.E. Glinsky et al., "Modeling of endovascular patch welding using the computer program LATIS", Proceedings of Laser Tissue Interaction VI, Vol. 2391, p. 262, 1995; G.B. Zimmerman and W.L. Kruer, "Numerical Simulation of laser initiated fusion", Controlled Fusion, Vol. 11, p. 82, 1975; R.A. London et al., "Laser-tissue interaction modeling with LATIS", submitted to Appl. Optics, 1997.

6. R. More et al., "A new quotidian equation of state (QEOS) for hot dense matter", Phys. Fluids, Vol. 31, p. 3059, 1988.

7. L. Haar, J.S. Gallagher and G.S. Kell, NBS/NRC Steam Tables, McGraw-Hill, 1984.

8. P. Amendt et al., "Modeling of bubble dynamics in relation to medical applications", Proceedings of Laser Tissue Interaction VIII, Vol. 2975, 1997.

9. H. Kull, "Theory of the Rayleigh-Taylor instability", Phys. Reports, Vol. 206, p. 197, 1991. 


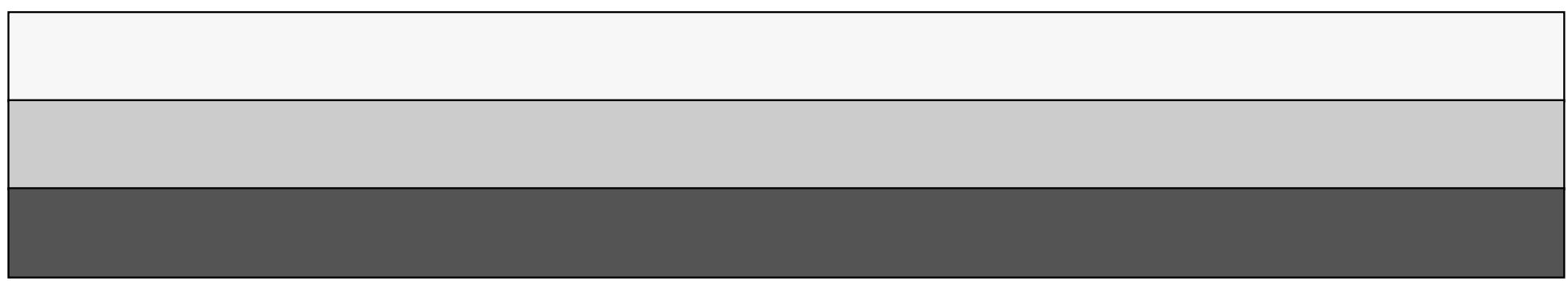

